\title{
FILLING POLYGONAL HOLES WITH BICUBIC PATCHES
}

John A. Gregory and Jianwel Zhou 



\title{
FILLING POLYGONAL HOLES WITH BICUBIC PATCHES
}

\author{
John A. Gregory and Jianwei Zhou
}

Department of Mathematics and Statistics

Brunei University, Uxbridge, UB8 3PH England

\begin{abstract}
Consider a bicubic rectangular patch complex which surrounds an $n$-sided hole in $\mathrm{R}^{3}$. Then the problem of filling the hole with $n$ bicubic rectangular patches is studied.
\end{abstract}

Keywords Bicubic patch, polygonal hole 
BRUNEL UNIVERSITY

SEP 1991

w9198831

LIBRARY 


\section{Introduction}

The problem of filling a polygonal hole, which occurs within a smooth parametric rectangular patch complex, is one which arises frequently in free-form surface modelling, for example, where a number of surfaces are to be blended together. Here, we consider the situation where the rectangular patch complex is composed of bicubic patches which form a $C^{1}$ surface about an n-sided hole. A method is proposed for filling the $n$-sided hole with $n$ bicubic rectangular patches such that the resulting patch complex is a $C^{1}$ surface.

The development of such a method is not new, for example [Van Wijk '86] gives a detailed study of such a technique, based on the use of a certain type of continuity constraint between the bicubic patches. However, [Liu '86], [Liu and Hoschek '89], and [Peters'89] have observed that a more general type of continuity constraint between polynomial patches is allowable, and it is the general continuity constraint condition which we propose to study here.

The bicubic method proposed here is that reported on in the tutorial paper [Gregory, Lau and Zhou '90], where a variety of techniques for filling $n$-sided holes is surveyed. In the case $n=3$, a closed form solution for filling the hole with three bicubic patches is produced. In the case of an $n$-sided hole, $n>4$, a solution is produced by constraining certain of the boundary data coefficients.

A Hermite representation of the bicubic patches will be assumed in the development of the theory, rather than the use of the Bernstein-Bézier representation. This will allow us to impose $C^{1}$ continuity constraints on the rectangular patch complex surrounding the hole through the choice of common Hermite data coefficients and this leads to some simplification of the analysis.

In section 2 we give a precise statement of the polygonal hole problem and introduce the basic continuity constraint conditions for filling the hole with bicubic patches. A detailed analysis of the continuity constraints is given in section 3. This analysis is used in the development of the schemes for filling an $n$-sided hole in the cases $n=3$ and $n>4$ described in section 4 . 


\section{Description of the Problem}

\subsection{Bicubic patch}

The bicubic Hermite patch $p:[0,1]^{2} \rightarrow \mathbb{R}^{3}$ is defined by

$$
p(u, v)=\left[H_{0}(u), H_{1}(u), H_{2}(u), H_{3}(u)\right] Q\left[H_{0}(v), H_{1}(v), H_{2}(v) H_{3}(v)\right]^{T},(u, v) \in[0,1]^{2}
$$

Where

$$
Q=\left[\begin{array}{cccc}
p_{0,0} & p_{0,1} & p_{0,0}^{v} & p_{0,1}^{v} \\
p_{1,0} & p_{1,1} & p_{1,0}^{v} & p_{1,1}^{v} \\
p_{0,0}^{u} & p_{0,1}^{u} & p_{0,0}^{u, v} & p_{0,1}^{u, v} \\
p_{1,0}^{u} & p_{1,1}^{u} & p_{1,0}^{u, v} & p_{1,1}^{u, v}
\end{array}\right]
$$

and

$$
\left.\begin{array}{l}
H_{1}(u)=-2 u^{3}+3 u^{2}=H_{0}(1-u), \\
H_{3}(u)=u^{3}-u^{2}=-H_{2}(1-u) \cdot
\end{array}\right\}
$$

This Hermite representation of a bicubic patch has the interpolation property that, at the vertices $(i, j)$ of $[0,1]^{2}$

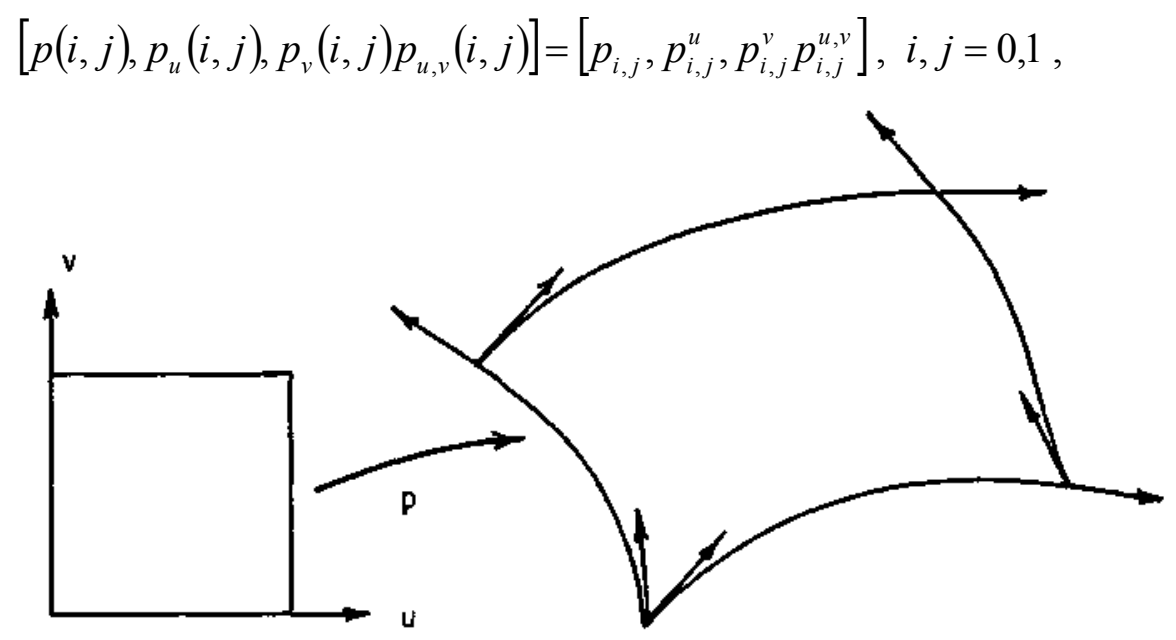

Figure 2.1 Bicubic Hermite Patch

where $p_{u}=\partial p / \partial u, p_{v}=\partial p / \partial v, p_{u v}=\partial^{2} p / \partial u \partial v$, see figure 2.1. Furthermore, along the edges, the 
boundary curves $p(u, 0), p(u, 1), p(0, v), p(1, v)$ and the cross boundary tangent vector derivatives $P_{v}(\mathrm{u}, 0)$, $p_{v}(u, 1), p_{u}(0, v), p_{u}(1, v)$ are univariate cubic Hermite functions. These univariate functions are determined completely by the vertex values on those edges. Thus two patches $p$ and $q$ can be joined with position and tangent plane continuity by appropriate identification of vertex data along their common boundary. This situation is well known in the construction of surface complexes of rectangular patches, where at each vertex there are four edges meeting (a "regular" vertex). The patch complex can then be considered as a single map from a parametric domain, subdivided by a regular rectangular mesh. We now wish to consider the more complex situation of filling an $n$-sided hole with $n$ bicubic patches, where there are now $n$ edges meeting at a vertex, $n \neq 4$ (a "non-regular" vertex).

\subsection{The polygonal hole problem}

Consider the situation shown in figure 2.2 of an $n$-sided "hole" in $\mathrm{R}^{3}$ surrounded by rectangular bicubic patches with regular vertices. The patches are assumed to form a $\mathrm{C}^{1}$ surface around the hole,

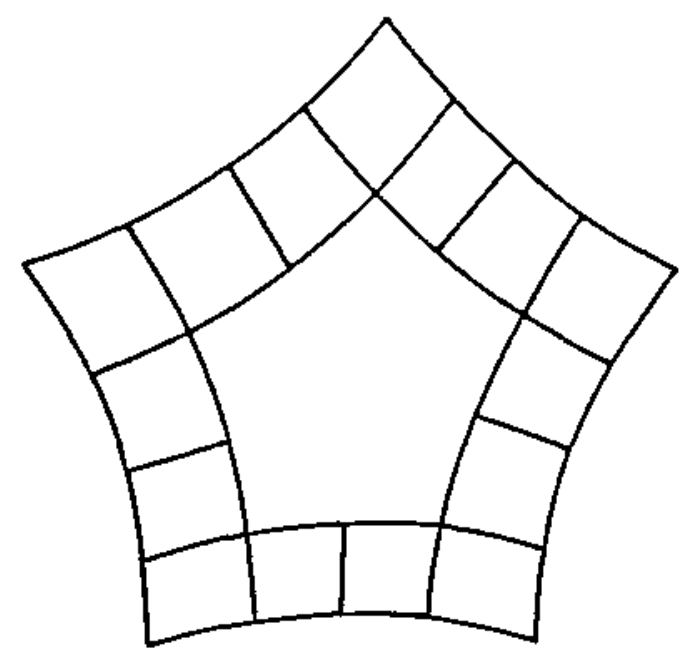

Figure 2.2 The polygonal hole problem

this being achieved by having identical vertex interpolation data along common edges. The $j$ th "boundary edge" of the hole, $j=0, \ldots, n-1$, is defined by two adjoining bicubic patches, as shown in the figure. (This includes the situation of having one bicubic patch adjacent to the boundary edge as a degenerate case.)

We wish to fill the $\mathrm{n}$-sided hole with $n$ bicubic patches $\boldsymbol{p}_{j}:[0,1]^{2} \rightarrow \mathbb{R}^{3}, j=0, \ldots, \mathrm{n}-1$, which meet at a non-regular $n$-vertex and which adjoin the regular rectangular patch complex with parameterizations as shown in figure 2.3. Thus $\boldsymbol{P} j(\mathrm{~s}, 1)$ and $\boldsymbol{P} j+1(1, \mathrm{~s})$ must match the $j$ ' th boundary edge of the hole and 


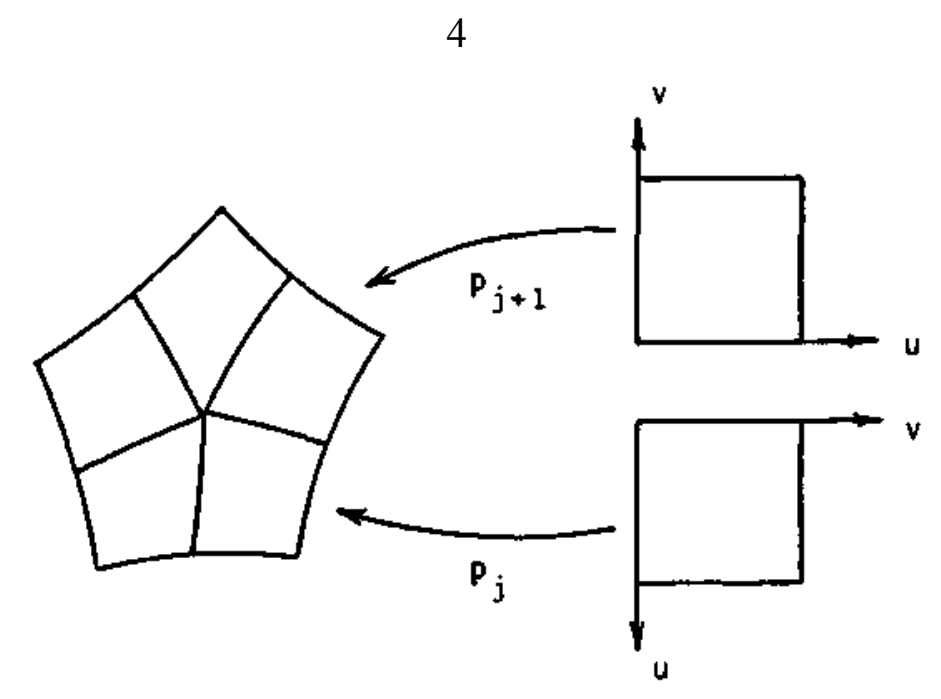

Figure 2.3 Filling the hole with rectangular patches

$\partial_{0,1} p j(s, 1)$ and $\partial_{1,0} \mathrm{pj}(1, \mathrm{~s})$ must match the cross boundary tangent. Hence the vertex data of the patches $\boldsymbol{p} j$ and $\boldsymbol{p} j_{+1}$ along the $j^{\prime}$ th edge of the hole are identified with those of the adjoining bicubic patches. In particular, we denote

$$
\left.\begin{array}{l}
B_{j}=p_{j}(0,1)=p_{j+1}(1,0) \\
B_{j}^{u}=-\partial_{1,0} p_{j}(0,1)=\partial_{0,1} p_{j+1}(1,0) \\
B_{j}^{v}=-\partial_{0,1} p_{j}(0,1)-\partial_{1,0} p_{j+1}(1,0) \\
B_{j}^{u, v}=\partial_{1,1} p_{j}(0,1)=-\partial_{1,1} p_{j+1}(1,0) .
\end{array}\right\}
$$

Similarly, the vertex data at the corners of the polygonal hole are also defined by the adjoining rectangular patches, but will not be required explicitly in the analysis. This leaves us with the degrees of freedom at the $n$-vertex, where we denote

$$
\left.\begin{array}{l}
Q \quad=p_{j}(0,0)=p_{j+1}(0,0) . \\
Q_{j}-\partial_{0,1} p_{j}(0,0)=\partial_{1,0} p_{j+1}(0,0), \\
Q_{j-1, j}=\partial_{1,1} p_{j}(0,0) .
\end{array}\right\}
$$

\subsection{The basic continuity constraint equations}

The choice of the bicubic Hermite data for the patches $\boldsymbol{p} j, j=0, \ldots, n-1$, of the previous subsection, means that these patches have $\boldsymbol{C}^{\mathbf{1}}$ joins with the bicubic patches surrounding the hole. We are 
thus concerned with achieving a $\mathrm{C}^{1}$ surface across the "interior edges" about the $n$-vertex $Q$. It is well known that this involves constraint equations of the form

$$
\begin{gathered}
P_{j+1}(s .0)-P_{\mathrm{j}}(0 . s)=0 \\
\alpha_{j}(s) \partial_{1,0} p_{j}(0, s)+\beta_{j}(s) \partial_{0,1} p_{j}(0, s)+\gamma_{j}(s) \partial_{0,1} p_{j+1}(s, 0)=0, j=0, \ldots ., n-1
\end{gathered}
$$

where

$$
\alpha_{j}(s) \gamma_{j}(s)>0
$$

The constraint (2.7) is that for $C^{\circ}$ continuity and in our case is automatically satisfied since $p_{j+1}(\mathrm{~s}, 0)$ and $p_{j+1}(0, \mathrm{~s})$ are univariate Hermite functions which share common interpolation data, see (2.5) (2.6). The constraint (2.8) is that for $C^{1}$, that is tangent plane, continuity and has become known as the "geometric" $G C^{1}$ constraint in the CAGD literature. The condition (2.9) is imposed to avoid cusp like joins between the patches.

In references [Liu '86], [Liu and Hoschek '89] and [Peters '89] it is observed that, in the case of bicubic patches, where $\partial_{1,0} p_{j}(0, s)$ and $\partial_{1,0} p_{j+1}(0, s)$ are cubic polynomials and $\partial_{1,0} p_{j}(0, s)$ is quadratic, the scalar factors $\alpha_{j}(s)$ and $\gamma_{j}(s)$ can be quintic polynomials and the $\beta_{j}(s)$ can be sextic. We consider this situation in detail in the following section. In particular, we are concerned with analysing the constraints (2.8) around the $n$-vertex.

\section{A Study of the Continuity Constraints}

\section{1 $\quad C^{1}$ continuity at the $n$-vertex}

We first consider the constraint equation (2.8) at the $n$-vertex $\boldsymbol{Q}$, where $s=0$. Thus with $s=0$ in (2.8) and (2.9) we obtain

$$
\alpha_{j}(0) Q_{j-1}+\beta_{j}(0) Q_{j}+\gamma_{j}(0) Q_{j+1}=0, j=0 \ldots, n-1
$$

Where

$$
\alpha_{j}(0) \gamma_{j}(0)>0
$$

It is required that

$$
\operatorname{dim} \operatorname{span}\left\{Q_{j}\right\}_{j=0}^{n}=2
$$


so that the vectors $\left\{\boldsymbol{Q}_{j}\right\}$ lie in a common tangent plane at $\boldsymbol{Q}$, see figure 3.1. We also assume, without loss of generality, that

$$
\alpha_{j}(0)=1, j=0, \ldots, n-1
$$

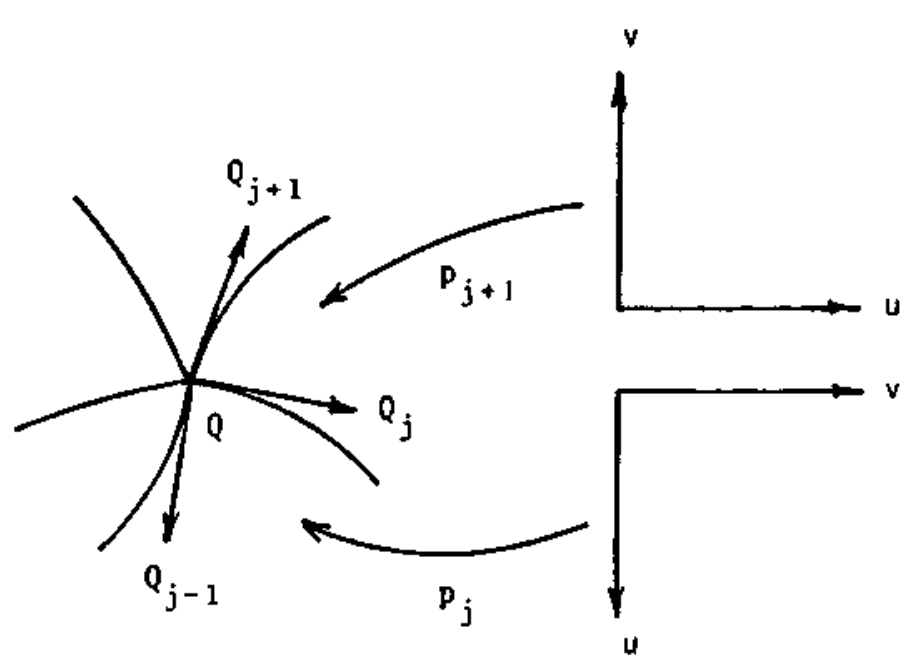

Figure 3.1 The $n$-vertex situation

In order to proceed with the analysis, we now consider the "symmetric" equal coefficient case, where

$$
\beta_{j}(0)=\beta_{0} \text { and } \gamma_{j}(0)=\gamma_{0}>0, j=0, \ldots, n-1
$$

The vertex constraints (3.1) are thus

$$
Q_{j-1}+\beta_{0} Q_{j}+\gamma_{0} Q_{j+1}=0, j=0, \ldots, n-1 .
$$

Furthermore, the following geometrical constraints on the vectors $\left\{Q_{j}\right\}_{j=0}^{n-1}$ are imposed: Let $\theta_{j}$ denote the angle from $\boldsymbol{Q}_{j}$ to $\boldsymbol{Q}_{j+1, j}=0, \ldots . n-1$ (all measured under a common orientation). Then it is assumed that

$$
\sum_{j=0}^{n-1} \theta_{j}=2 \pi \text { and } \theta_{j}>0, j=0, \ldots ., n-1
$$


These assumptions imply that the vectors $\left\{\boldsymbol{Q}_{i}\right\}$ form a non-overlapping star in the tangent plane at $\boldsymbol{Q}$ and that

$$
Q_{j} \neq Q_{\ell} \text { for } j \neq \ell, 0 \leq j, \ell \leq n-1,
$$

see figure 3.1. We now have the following:

Proposition 3.1. There exists $\left\{Q_{j}\right\}_{j=10}^{n-1}$ satisfying the vertex constraints (3.6) and the non-overlapping star condition (3.7) if and only if

$$
\beta_{0}=-2 \cos (2 \pi / n) \text { and } \gamma_{0}=1 \text {. }
$$

Furthermore,

$$
\left.Q_{j}=\mid \sin (j 2 \pi / n) Q_{1}-\sin (j-1) 2 \pi / n\right) Q_{0} V \sin (2 \pi / n), j-0, \ldots . n-1 .
$$

Proof. The constraints (3.6) define a homogeneous, constant coefficient, difference equation giving

$$
Q_{1}=\left(Q_{1}-\mu Q_{0}^{j}\right) \lambda+\left(\lambda Q_{0}-Q_{1}\right) \mu^{j} V(\lambda-\mu) \text { for } \lambda \neq \mu,
$$

and

$$
Q_{j}=\left|Q_{0}+\left(Q_{1} \lambda-Q_{0}\right) j\right| \lambda^{j} \quad \lambda=\mu
$$

where $\lambda, \mu$ are the roots of the auxillary equation

$$
1+\beta_{0} m+\gamma_{0} m^{2}=0
$$

Hence

$$
\lambda, \mu=1 / \gamma_{0} \text { and } \lambda+\mu=-\beta_{0} / \gamma_{0} .
$$

The solution (3.12) cannot satisfy the periodicity requirement $Q_{0}=Q_{n}$ whilst maintaining the linear independence of $Q_{0}$ and $Q_{1}$ Thus (3.11) is the only allowable solution and imposing periodicity gives $\lambda^{\mathrm{n}}=\mu^{\mathrm{n}}=1$, which implies that

$$
\lambda=e^{i k 2 \pi / n}, \mu=e^{i \ell 2 \pi / n}, 0 \leq k, \ell \leq n-1 .
$$

The requirement that $\lambda \mu$ and $\lambda+\mu$ be real (see (3.14)) then leads, after further analysis, to the restriction that 


$$
\lambda=e^{i k 2 \pi / n}, \mu=e^{-i k 2 \pi / n}, 1 \leq k<n / 2 .
$$

Hence

$$
Q_{j}=\left[\sin \left(j \varphi_{k}\right) Q_{1}-\sin (j-1) \varphi_{k}\right) Q_{0} V \sin \varphi_{k}, j=0, \ldots, n-1
$$

Where

$$
\varphi_{k}=2 \pi k / n \in(0, \pi) \text { for } 1 \leq k<n / 2 \text {, }
$$

and thus $\sin \varphi_{k}>0$ We now consider the case $2 \leq k<n / 2$. In this case there exists $\ell$, with $3 \leq \ell \leq \mathrm{n}-1$, such that $(\ell-1) k<n \leq \ell k$. If $n=\ell k$ then (3.15) gives $\mathrm{Q}_{\ell}=\mathrm{Q}_{0}$ which contradicts (3.8). Thus $(\ell-1) k<n<\ell k$, that is $0<\ell k-n<k$. Therefore

$$
\left.\ell \varphi_{k}=2 \pi+2(\ell k-n) \pi\right) \pi \in(2 \pi .3 \pi)
$$

and

$$
(\ell-1) \varphi_{k}=2 \pi+2(\ell k-n-k) \pi / n \in(\pi, 2 \pi) .
$$

Hence

$$
\sin \left(1 \varphi_{k}\right)>0 \text { and } \sin \left((1-1) \varphi_{k}\right)<0
$$

Thus, from (3.15), $Q_{\ell}$ lies between $Q_{0}$ and $Q_{1}$ since it is a positive linear combination of $\mathrm{Q}_{0}$ and $\mathrm{Q}_{\mathrm{t}}$. This violates (3.7). Hence $k=1$ is the only value acceptable in (3.15) which completes the proof.

Remark 3.2. A corollary to Proposition 3.1, which follows from (3.3), is that the rank of the cyclic coefficient matrix defined in (3.6) is $n-2$.

\section{2 $\quad C^{1}$ continuity across the interior edges}

We now study the continuity constraint equations (2.8) in the symmetric case where

$(s)$,

and ( ) are independent of $j$. Thus (2.8) is written as

$$
\alpha(s) \partial_{1,0} p_{j}(0, s)+\beta(s) \partial_{0,1} p_{j}(0, s)+\gamma(s) \partial_{0,1} p_{j+l}(s, 0)=0
$$

where we denote the scalar polynomials by

$$
\begin{array}{ccc}
\alpha(s)=\sum_{i=0}^{\infty} \alpha_{i} s^{i}, \beta(s)=\sum_{i=0}^{\infty} \beta_{i} s^{i}, \gamma(s)=\sum_{i=0}^{\infty} \gamma_{i} s^{i} . \\
i=0 & i=0 & i=0
\end{array}
$$


(The suffix notation in (3.17) should not be confused with that for $\alpha_{j}(0), \beta_{j}(0)$, and $\gamma_{j}(0)$ in subsection 3.1.) It was observed in section 2 that $\alpha, \gamma$ can be at most quintic polynomials and $\beta$ can be at most sextic but for our purposes here it is easier to assume the general power series forms (3.17), where the higher order coefficients are zero. Our purpose is to analyse the solvability of (3.16) assuming that the polygonal hole data (2.5) are given a priori. We will see that in the case $n=3$ a solution of (3.16) is possible but that for $n \geq 5$ a solution is not possible, in general. In section 4 we will consider a specific solution for the case $n=3$. In the case $n \geq 5$ we will obtain a solution of (3.16), where some of the polygonal hole data (2.5) are determined a posteriori.

Equating coefficients in (3.16) gives

$$
\begin{aligned}
E_{i, j}:=6 & \left(\beta_{i-1}-\beta_{i-2}\right)(B-Q)+\left(\alpha_{i}-3 \alpha_{i-2}+2 \alpha_{i-3}\right) Q_{j-1} \\
& +\left(\beta_{i}-4 \beta_{i-1}+3 \beta_{i-2}\right) Q_{j}+\left(\gamma_{i}-3 \gamma_{i-2}+2 \gamma_{i-3}\right) Q_{j+1} \\
& -\left(3 \alpha_{i-2)}-2 \alpha_{i-3}-3 \gamma_{i-2}+2 \gamma_{i-3}\right) B_{j}^{u}+\left(2 \beta_{i-1}-3 \beta_{i-2}\right) B_{j}^{v} \\
& +\left(\alpha_{i-1}-2 \alpha_{i-2}+\alpha_{i-3}\right) Q_{j-1, j}+\left(\gamma_{i-1}-2 \gamma_{i-2}+\gamma_{i-3}\right) Q_{j, j+1} \\
& -\left(\alpha_{i-2}-\alpha_{i-3}-\gamma_{i-2}+\gamma_{i-3}\right) B_{j}^{u, v}=0, i=0,1, \ldots .
\end{aligned}
$$

where any coefficients with negative suffices are interpreted as zero. For $i=0,(3.18)$ gives the vertex constraint

$$
\alpha_{0} Q_{j-1}+\beta_{0} Q_{j}+\gamma_{0} Q_{j+1}=0, j=0, \ldots n-1
$$

which from subsection 3.1 has the solution

$$
\alpha_{0}=1=\gamma_{0}, \beta_{0}=-2 \cos (2 \pi / n) .
$$

For the purposes of analysis we consider the transformed equations

$$
\sum_{i=0}^{\infty} i^{k} E_{i, j}=0, \quad j=0, \ldots . . n-1
$$

for $k=0,1, \ldots$ where $E_{i, j}$ is defined by (3.18).

We then make the following definition 
Definition 3.3. We say that the constraints (3.21) are removable if for arbitrarily given boundary data $\left\{B_{j}, B_{j}^{u}, B_{j}^{v}\right\}_{j=0}^{n-1}$ there exists scalars $\left\{\alpha_{\mathrm{i}} \beta_{\mathrm{i}}, \gamma_{\mathrm{i}}\right\}_{\text {and }}$ vectors $Q\left\{Q_{j} Q_{j, j+1}\right\}_{j=0}^{n-1}$ such that the constraints are satisfied.

Proposition 3.4. For $n \geq 5$ the constraints (3.21) are removable if and only if

$$
\alpha(s)=\beta(s)=\gamma(s) \equiv 0 .
$$

Proof We first consider the constraint

$$
\sum_{i} E_{i j} \equiv-\sum_{i}\left(\alpha_{i}-\gamma_{i}\right) B_{j}^{u}-\sum_{i} B_{j}^{v}=0
$$

Then

$$
\sum_{i}\left(\alpha_{i}-\gamma_{i}\right)=0=\sum_{i} \beta_{i}
$$

since the two tangent vectors $B_{j}^{u}$ and $B_{j}^{v}$ at the boundary vertex $B_{j}$ must be linearly independent. We now consider

$$
\sum i E_{i, j}=0
$$

which, using (3.24), gives

$$
-\sum i\left(\alpha_{i}-\gamma_{i}\right) B_{j}^{u}-\sum i \beta_{i} B_{j}^{v}=0 .
$$

Hence

$$
\sum i\left(\alpha_{i}-\gamma_{i}\right)=0=\sum i \beta_{i} .
$$

We now proceed by induction, assuming that the constraints (3.21) are removable for $k=0, \ldots, m$, $m \geq 1$, if and only if

$$
\begin{gathered}
\sum i^{k} \alpha_{i}=0, k=0, \ldots ., m-2, \\
\sum i^{k} \beta_{i}=0=\sum i^{k}\left(\alpha_{i}-\gamma_{i}\right), \quad i=0, \ldots . . m .
\end{gathered}
$$

(In the case $m=1,(3.27)$ is void.) (3.21) for $\mathrm{k}=m+1$ gives 


$$
6 \beta_{0} \sigma_{1, m} Q_{j}+\sigma_{2, m}^{u} B_{j}+\sigma_{3, m}^{v} B_{j}=2 \sigma_{1, m}\left(Q_{j-1, j}+Q_{j, j+1}\right)
$$

where

$$
\sigma_{1, m}=\left(\begin{array}{c}
m+1 \\
2
\end{array}\right) \sum i^{m-1} \alpha_{i}, \sigma_{2, m}=\sum i^{m+1}\left(\alpha_{i}-\gamma_{i}\right), \sigma_{3, m}=\sum i^{m+1} \beta_{i}
$$

Eliminating the term $Q_{j-1, j}+Q_{j, j+1}$ by use of (3.18) for $\mathrm{i}=1$ then gives

$$
\begin{aligned}
12 \beta_{0} \sigma_{1, m}\left(B_{j}-Q\right)+ & 2 \sigma_{1, m}\left(\alpha_{1} Q_{j-1}+\left(\beta_{1}-\beta_{0}\right) Q_{j}+\gamma_{1} Q_{j+1}\right) \\
& +\sigma_{2, m} B_{j}^{u}+\left(\sigma_{3, m}+4 \beta_{0} \sigma_{1, m}\right) B_{j}^{v}=0, j=0, \ldots ., n-1
\end{aligned}
$$

Taking a linear combination of equations (3.31) for $j=i-1$ and $i+1$ together with a $\beta_{0}$ multiple for $j=i$ and using the vertex constraints

$$
Q_{j-1}+\beta_{0} Q_{j}+Q_{j+1}=0, \quad j=0, \ldots . n-1
$$

gives

$$
\begin{aligned}
12 \beta_{0} \sigma_{1, m}\left(B_{i-1}\right. & \left.+\beta_{0} B_{1}+B_{i+1}-\left(2+\beta_{0}\right) Q\right)+\sigma_{2, m}\left(B_{i-1}^{u}+\beta_{0} B_{i}^{u}+B_{i+1}^{u}\right) \\
& +\left(\sigma_{3, m}+4 \beta_{0} \sigma_{1, m}\right)\left(B_{i-1}^{v}+\beta_{0}^{v} B_{i}+B_{i+1}^{v}\right)=0, i=0, \ldots n-1 .
\end{aligned}
$$

Taking the difference of (3.32) for $i=\ell$ and $i=\ell+1$ then gives

$$
\begin{aligned}
& 12 \beta_{0} \sigma_{1, m}\left[\left(B_{\ell-1}-B_{\ell}\right)+\beta_{0}\left(B_{\ell}-B_{\ell+1)}+\left(B_{\ell+1}-B_{\ell+2}\right)\right]\right. \\
& +\sigma_{2, m}\left[\left(B_{\ell-1}^{u}-B_{\ell}^{u}\right)+\beta_{0}\left(B_{\ell}^{u}-B_{\ell+1}^{u}\right)+\left(B_{\ell+1}^{u}-B_{\ell+2}^{u}\right)\right] \\
& +\left(\sigma_{3, m}+4 \beta_{0} \sigma_{1, m}\right)\left[\left(B_{\ell-1}^{v}-B_{\ell}^{v}\right)+\beta_{0}\left(B_{\ell}^{\ell}-B_{\ell+1}^{v}\right)+\left(B_{\ell+1}^{v}-B_{\ell+1}^{v}\right)\right]=0 .
\end{aligned}
$$

For $n \geq 5$ the three vectors in (3.33) will, in general, be linearly independent and $\beta_{0} \neq 0$ for $n \neq 4$, see (3.9). Hence

$$
\sigma_{1, \mathrm{~m}}=\sigma_{2, \mathrm{~m}}=\sigma_{3, \mathrm{~m}}
$$

which completes the inductive step. 
Remark 3.5 From Proposition 3.4 we conclude that the continuity constraints are not removable in general for $n \leq 5$ and hence we cannot assume arbitrarily given Hermite boundary data in this case. For $n=3$, however, equation (3.33) is satisfied identically since $\beta_{0}=1$ and (3.32) gives an identical equation for $j=0,1,2$, In this case the constraints are removable.

This completes the analysis of the continuity constraints and we are now in a position to develop the special solutions for the polygonal hole problem.

\section{Methods for Filling the n-sided Hole}

In this section we consider particular solutions to the continuity constraint equaitons (3.16) which result in practical methods for filling the $n$-sided hole with bicubic patches. As was observed in the previous section, we find it appropriate to distinguish between the case $n=3$ and the general case $n \geq 5$. We first, however, consider the choice of the scalar coefficients $\alpha(s), \beta(s)$ and $\gamma(s)$ in the constraints (3.16).

\subsection{A particular continuity constraint}

From (3.9) we have

$$
\alpha(0)=1=\gamma(0) \text { and } \beta(0)=-2 \cos (2 \pi / \mathrm{n})
$$

Also, from (3.24) and (3.26) we see that

$$
\beta(1)=\beta^{\prime}(1)=0 \text { and } \alpha(1)-\gamma(1)=\alpha^{\prime}(1)-\gamma^{\prime}(1)=0 \text {. }
$$

This latter condition reflects the fact that $B_{j}^{u}$ and $B_{j}^{v} \quad$ must be linearly independent for a regular surface. For simplicity, we now choose the minimum degree scalar polynomials consistent with (4.1) and (4.2), namely,

$$
\alpha(s)=\gamma(s)=1 \text { and } \beta(s)=\beta_{0}(1-s)^{2}
$$

where

$$
\beta_{0}=-2 \cos (2 \pi / n)
$$

Substituting in (3.16) then gives the quartic polynomial equation 


$$
\varphi_{j}(s)=\partial_{1,0} p_{j}(0, s)-2(1-s)^{2} \cos (2 \pi / n) \partial_{1,0} p_{j}(0, s)+\partial_{0,1} p_{j+1}(0, s)=0,
$$

where, by the choice (4.3),

$$
\varphi_{j}(0)=\varphi_{j}(1)=\varphi^{\prime}{ }_{j}(1)=0 \text {. }
$$

Thus, we only require two additional constraints in order that (4.5) is satisfied. We take these as

$$
\varphi^{\prime}{ }_{j}(0)=\varphi^{\prime \prime}{ }_{j}(0)=0
$$

which gives the two constraints

$$
\left\{\begin{array}{l}
6 \beta_{0}\left(B_{j}-Q\right)-6 \beta_{0} Q_{j}+2 \beta_{0} B_{j}^{v}+Q_{j-1, j}+Q_{j, j+1}=0, \\
-18 \beta_{0}\left(B_{j}-Q\right)+15 \beta_{0} Q_{j}-7 \beta_{0} B_{j}^{v}-2 Q_{j-1, j}-2 Q_{j, j+1}=0, j=0, \ldots, n-1,
\end{array}\right.
$$

see equations (3.18) for $i=1$ and 2. Thus, for the choice of the scalar functions (4.3), the constraints (4.6) are necessary and sufficient conditions for a $G C^{1}$ join between the patches $p_{j}$ and $P_{j+1}$. To these conditions we must also add the vertex constraint

$$
Q_{j-1}+\beta_{0} Q j+Q_{j+1}=0 \quad j=0, \ldots, n-1,
$$

see (3.19) and (3.20).

\subsection{Solution for the case $n=3$}

In the case $n=3$ we have $\beta_{0}=1$ and the constraints (4.6) and (4.7) then give

$$
\left\{\begin{array}{l}
6\left(B_{j}-Q\right)-6 Q_{j}+2 B_{j}^{v}=-\left(Q_{j-1, j}+Q_{j, j+1}\right) \\
2\left(B_{j}-Q\right)-Q_{j}+B_{j}^{v}=0, \quad j=0,1,2 \\
Q_{0}+Q_{1}+Q_{2}=0
\end{array}\right.
$$

We then have

Proposition 4,1 A symmetric solution to the triangular hole problem is

$$
\left\{\begin{array}{l}
Q \quad=\frac{1}{3} V+\frac{1}{6} W, \\
Q_{j}=2 B_{j}+B_{j}^{v}-\frac{2}{3} V-\frac{1}{3} W, \quad j=0,1,2 \\
Q_{j-1, j+1}=-6 B_{j}-4 B_{j}^{v}+2 V+\frac{3}{2} W,
\end{array}\right.
$$


where

$$
V=B_{0}+B_{1}+B_{2}, W=B_{0}^{v}+B_{1}^{v}+B_{2}^{v}
$$

Here, the value of $Q$ in (4.9) follows by summing the second equation in (4.8) over $j$ and this second equation is then solved for $Q_{j}$. The value of $Q_{j-1, j+1}$ is now given by the solution of the cyclic system defined by the first equation in (4.8) for $j=0,1,2$.

Remark 4.2 The above analysis shows that the continuity constraints are 'removable' in the case $n=3$, that is, there is a solution for arbitrarily given Hermite boundary data around the triangular hole.

Figures 4.1 - 4.3 show a model example of a triangular hole being filled with three rectangular patches with the interior vertex data determined by (4.9). It can be seen that a satisfactory tangent plane continuous surface is obtained by this method.

\subsection{Solution for general $\mathrm{n}$}

The analysis of section 3 shows that it is generally impossible to obtain a cubic Hermite patch solution to the polygonal hole problem in terms of arbitrarily given Hermite boundary data. In this case we can allow the Hermite boundary data parameters $\left(B j, B_{j}^{v}\right), j=0, \ldots, n-1$, to be additional degrees of freedom.

These parameters, together with the $n$-vertex parameters $Q$ and $\left(Q_{j}, Q_{j, j+1}\right), j=0, \ldots, n-1$, are then constrained by the underdetermined system of equations (4.6) and (4.7). There are then many possible ways of seeking appropriate solutions to this underdetermined system. For simplicity here, we observe that if the $n$-vertex parameters are given subject to the vertex constraint (4.7), then

$$
\left\{\begin{array}{l}
B_{j}=Q+2 Q_{j}-\frac{1}{2 \beta_{0}}\left(Q_{j-1, j}+Q_{j, j+1}\right) \\
B_{j}^{v}=-3 Q_{j}+\frac{1}{\beta_{0}}\left(Q_{j-1, j}+Q_{j, j+1}\right), j=0, \ldots, n-1,
\end{array}\right.
$$

provides a solution to the problem. However, figure 4.5 shows that this simple technique of perturbing some of the boundary data can lead to underirable 'bumps" being introduced into the tangent plane continuous surface. Here the pentagonal hole of figure 4.4 has been filled with five rectangular patches, where the interior vertex data has been determined by an averaging process and the boundary data has been perturbed to satisfy (4.11). 
Figure 4.6 shows the effect of partitioning the hole with an additional layer of bicubic patches adjacent to the boundary. This enables given Hermite boundary data to be matched exactly but introduces many additional degrees of freedom. The additional layer has thus been constrained by a simple splitting technique to reduce the number of degrees of freedom, see [Zhou '91] for further details. The remaining degrees of freedom have been chosen by trial and error to give the satisfactory solution of the figure.

\subsection{Conclusion}

The main purpose of this paper has been to give a detailed analysis of the continuity equations which result from constraining $n$ patches to meet at a non-regular $n$-vertex. For the case of a 3 -vertex, it has been shown that there is a simple closed form solution of the constraint equations which enables a

triangular hole to be filled with three bicubic patches. For the case of an $n$-vertex, $n \geq 5$, a solution of $n$-sided hole problem is produced by constraining certain of the boundary data. In practice, we feel that some variational surface criterion should be applied to the problem of determining an appropriate solution in this case, but have considered an investigation of such criteria to be beyond the scope of the present paper. However, the theory and examples presented here demonstrate the existence of tangent plane continuous bicubic patch methods for filling polygonal holes.

\section{Acknowledgements}

We gratefully acknowledge the support of the ACME Directorate of the Science and Engineering Research Council, with grants GR/E 25092 and GR/F 80838, and of Siemens AG, with a Ph.D. studentship.

\section{References}

Gregory, J.A., Lau, V.K.H. and Zhou, J. (1990), Smooth parametric surfaces and $n$-sided patches, in W. Dahmen, M. Gasca and C.A. Micchelli (eds.), Computation of Curves and Surfaces, NATO ASI

Series, Kluwer Academic Publishers, 457-498.

Liu, D. (1986), A geometric condition for smoothness between adjacent Bezier surface patches, Acta Mathematicae Applicatae Sinica 9, 432-442. 
Liu, D. and Hoschek, J. (1989), GC ${ }^{1}$ continuity conditions betweeen adjacent rectangular and triangular Bezier surface patches, Computer Aided Design 21, 194-200.

Peters, J. (1989), Local interpolation of a cubic curve mesh by a piecewise biquartic $\mathrm{C}^{1}$ surface without splitting, Tech. Report 89-25, University of Wisconsin.

Van Wijk, JJ. (1986), Bicubic patches for approximating non-rectangular control-point meshes, Computer Aided Geometric Design 3, 1-13.

Zhou, J. (1991), Geometric continuity and rectangular patch methods for surface modelling, PhD. thesis, Department of Mathematics and Statistics, Brunei University, in preparation. 


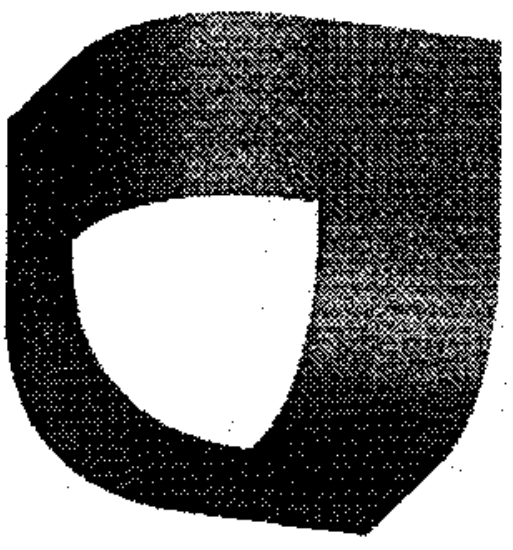

Figure 4.1

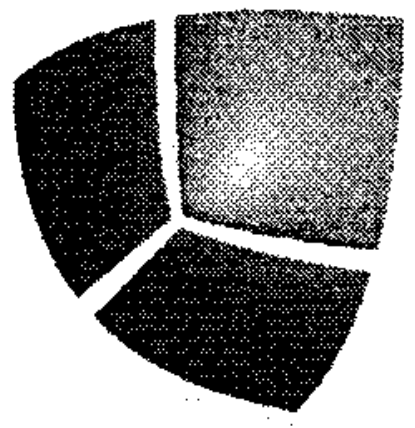

Figure 4.2

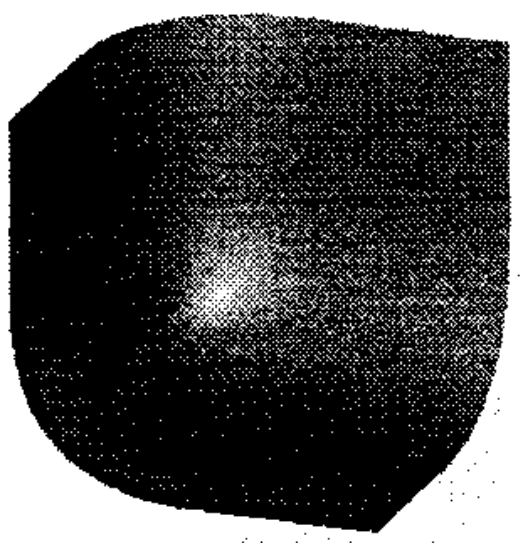

Figure 4.3 


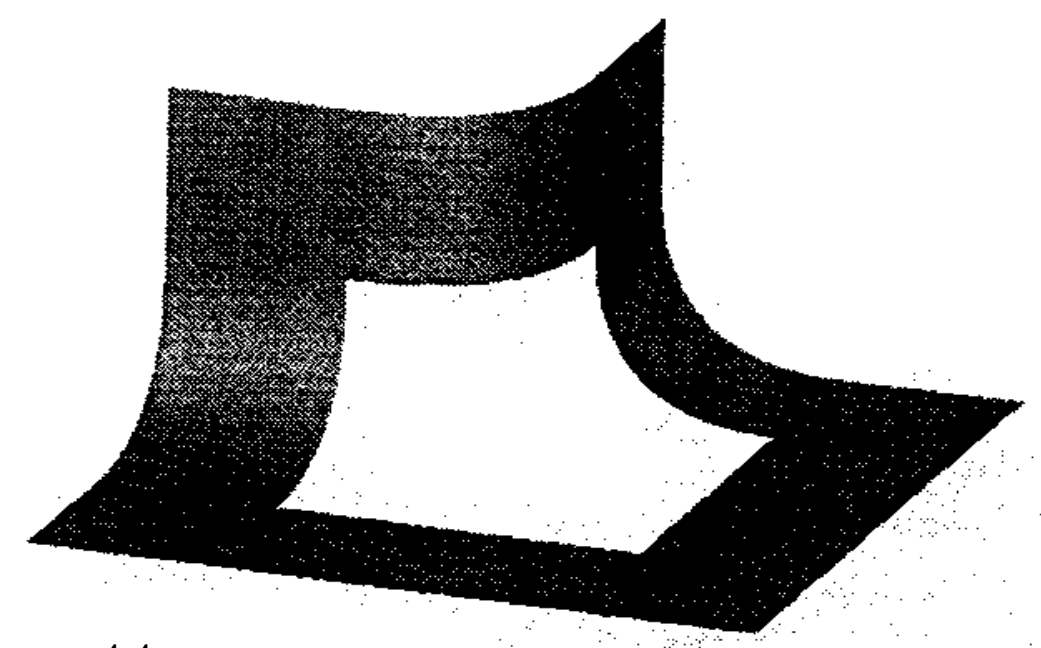

Figure 4.4

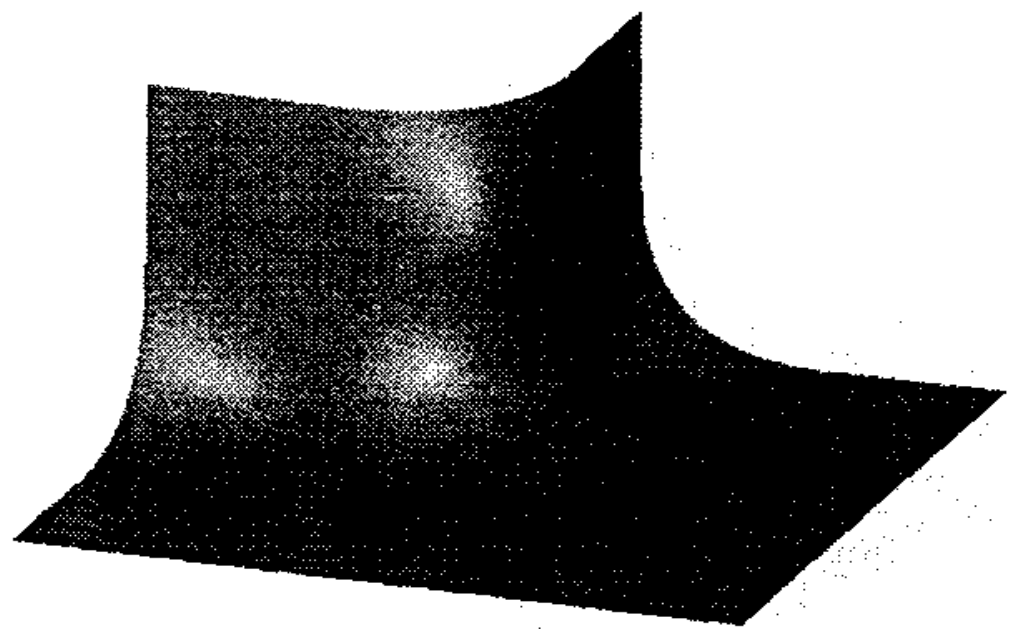

Figure 4.5

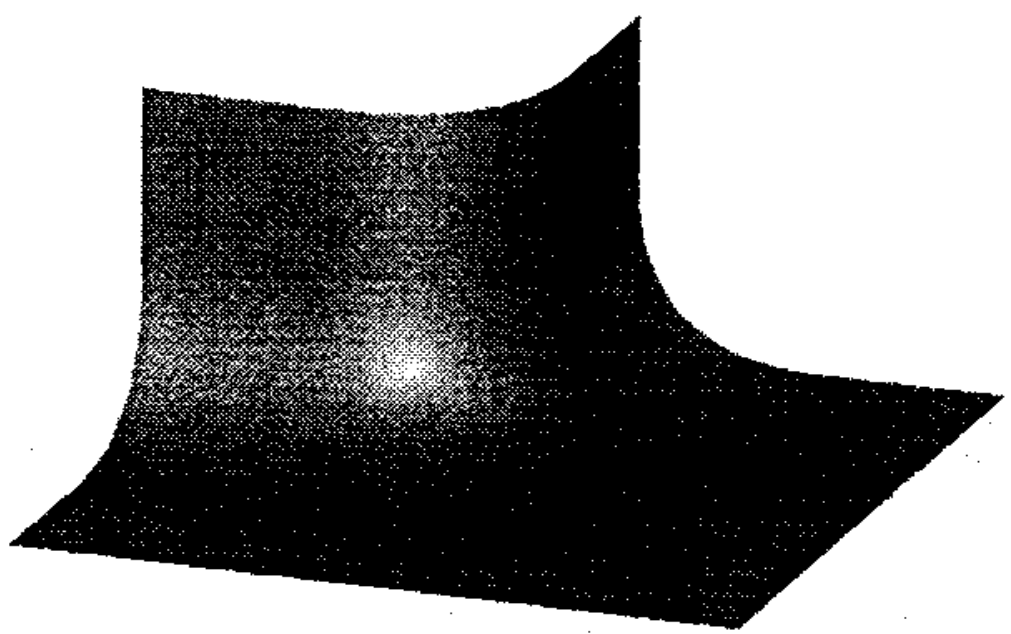

Figure 4.6

XB $2321421 X$

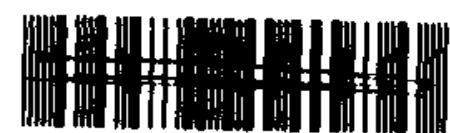

